throid short-term radioprotection requires a BMP4-dependent, selfrenewing population of stress erythroid progenitors. J Clin Invest. 2010;120(12):4507-4519.

10. Xiang J, Wu DC, Chen Y, Paulson RF. In vitro culture of stress erythroid progenitors identifies distinct progenitor populations and analogous human progenitors. Blood. 2015;125(11):1803-1812.

11. Flygare J, Rayon Estrada V, Shin C, Gupta S, Lodish HF. HIF1alpha synergizes with glucocorticoids to promote BFU-E progenitor selfrenewal. Blood. 2011;117(12):3435-3444

12. Hara H, Ogawa M. Erthropoietic precursors in mice with phenylhy- drazine-induced anemia. Am J Hematol. 1976;1(4):453-458.

13. Chyla BJ, Moreno-Miralles I, Steapleton MA, et al. Deletion of Mtg16, a target of $\mathrm{t}(16 ; 21)$, alters hematopoietic progenitor cell proliferation and lineage allocation. Mol Cell Biol. 2008;28(20):62346247.

14. Stadhouders R, Cico A, Stephen T, et al. Control of developmentally primed erythroid genes by combinatorial co-repressor actions. Nat Commun. 2015;6:8893.

15. Zhang J, Liu Y, Han X, et al. Rats provide a superior model of human stress erythropoiesis. Exp Hematol. 2019;78:21-34 e23.

\title{
COVID-19 and sickle cell disease
}

\section{Laurel A. Menapace and Swee Lay Thein}

Sickle Cell Branch, National Heart Lung and Blood Institute, National Institutes of Health, Bethesda, MD, USA

E-mail: SWEE LAYTHEIN - sl.thein@nih.gov

doi:10.3324/haematol.2020.255398

T he spread of the novel SARS-CoV-2 coronavirus (COVID-19) has resulted in widespread lockdowns and an unprecedented number of deaths globally.,2 The pandemic has posed unique challenges to healthcare providers involved in the care of individuals with chronic conditions, balancing maintenance of necessary care with appropriate precautions to reduce their exposure to infection. Patients with sickle cell disease (SCD) present multiple challenges due to the complexity of their condition, disease-related comorbidities, and need for frequent medical interventions. To date, there has been a paucity of published data on how COVID-19 may impact morbidity and mortality in SCD patients.

In this edition of Haematologica, Charkravorthy and colleagues report associated outcomes in 10 UK patients infected with SARS-CoV-2. ${ }^{3}$ All patients had hemoglobin $(\mathrm{Hb})$ SS disease with pre-existing co-morbidities, despite of this nine patients made a full recovery without receiving COVID-19 directed therapies. Five patients were hospitalized and treated supportively; two patients who had a cough and hypoxia received early preventive simple transfusions; five patients were managed at home via close telephone contact. Of the 10 patients, seven were female (median age of 37 years); two received hydroxyurea therapy and seven received regular blood transfusions (four exchange and three simple transfusions). Given that COVID-19 is an acute infectious pneumonia, most experts anticipated that SARS-CoV-2 infection would trigger acute chest syndrome (ACS) yet only one patient who died in this series had significant respiratory complications. The patient who died was a 54-year-old female with severe asthma, alloimmunization, and a history of delayed hemolytic transfusion reactions which prevented routine transfusion. The patient had lymphopenia, thrombocytopenia and elevated C-reactive protein (CRP), which have been identified as poor prognostic markers in COVID-19 patients. ${ }^{4}$ The authors acknowledge that the demographic and treatment characteristics of this cohort may have influenced the observed outcomes.

This edition also includes a report of 195 cases of suspected or confirmed SARS-CoV-2 infection as captured by 10 regional centers in a real time survey over a 4 -week period in the UK. ${ }^{5}$ Patients with SCD represented the majority of cases ( $\mathrm{n}=166,85.1 \%$ ) with $\mathrm{Hb}$ SS disease as the most common genotype (Hb SS $64.1 \%$; Hb SC $15.4 \%$; other genotypes $5.6 \%$ ) followed by thalassemia $(n=26$, $13.3 \%)$ and rare inherited anemias $(n=3,1.5 \%)$. While the incidence of ACS in the SCD cohort was not specified, red cell exchange was performed in 46 patients during the course of infection. A total of eight SCD patients required mechanical ventilatory support in the setting of respiratory failure; 11 of 13 deaths attributed to COVID-19 occurred in patients with SCD. A subset analysis of 76 hospitalized SCD patients with PCR confirmed SARSCoV-2 showed that the patient age was significantly associated with mortality, with the most deaths occurring in those aged 50 and older $(n=4)$. The authors noted that three patients aged 20-39 years in the subset succumbed to COVID-19 although details regarding the comorbid medical conditions were not provided. Unexpectedly, mortality was higher in females and was not associated with severity of disease but observed differences were insignificant. The cases reported to date represent $1.2 \%$ of the estimated 13,655 individuals with SCD per registry data in England. There was a low incidence of infection in children $(n=20)$ and there were no pediatric deaths. Definitive conclusions regarding COVID-19 mortality rates in SCD cannot be drawn given inherent ascertainment bias and missing data in the survey.

The mild clinical course of COVID-19 in the King's College Hospital SCD patients ${ }^{3}$ was similarly encountered in a case series of four patients from Chicago, ${ }^{6}$ all of whom presented with acute pain. Of the four patients, three received the usual supportive care but one patient, a 32-year-old male with Hb SS, developed ACS requiring intubation and exchange transfusion.

Two other centers reported COVID-19 pneumonia causing ACS in SCD patients. A 21-year-old male with $\mathrm{Hb} S / \beta^{\circ}$ thalassemia on hydroxyurea therapy presented with acute chronic left hip pain and subsequently developed a cough with progressive hypoxia and evidence of new pulmonary infiltrates during his hospital course.? SARS-CoV-2 PCR testing was positive during the second week of admission. In addition to antibiotic therapy, the patient was initiated 
Triage of SCD patients with Acute Symptoms of Fever, Cough, Dyspnea, Myalgia

or Atypical Symptoms* suggestive of COVID-19 Infection

Telephone Evaluation

- Symptoms mild, arrange testing via drive-through centers when feasible

- If no acute pain, $\mathrm{T}<38 \mathrm{C}, \mathrm{O}_{2}$ sat $>95 \%$ on room air, and no dyspnea, monitor at home

- +/- increased oral analgesia, +/antibiotics

- Patient should be instructed to selfisolate

- Patient to contact providers urgently if symptoms not improving, including development of dyspnea, high grade fever, severe pain, or other concerning symptoms
Symptoms Requiring On-site Medical Evaluation:

1. Acute pain requiring parenteral analgesia; 2 . $T>38 \mathrm{C} ; 3 . \mathrm{O}_{2}$ sat

$<94 \%$ on room air; 4 . Respiratory rate $>20$ or subjective dyspnea

\section{Inpatient Evaluation \& Treatment}

- Evaluate for ACS and provide standard of care, including blood transfusion if indicated.

- Obtain viral respiratory pathogen panel, CBC and differential, blood cultures, and chest x-ray.

- Initiate empiric antibiotic therapy within 60 minutes of arrival.

- Consider CT chest to assess for pathognomonic pulmonary findings of COVID-19, including ground glass opacities (GGO).

- Exclude SARS-CoV-2 infection with RT-PCR, repeat test if negative and high index of suspicion for COVID-19 using sputum sample.

- If COVID-19 confirmed, directed therapies including hydroxychloroquine** and antivirals should ONLY be provided in the context of a clinical trial.

- Thromboprophylaxis should be administered to patients without contraindications to anticoagulation. Monitor platelets, coagulation parameters, D-dimer, and fibrinogen levels.

Figure 1. Triage of managing individuals with Sickle cell disease in the CovID-19 pandemic. *Loss of smell (anosmia) and taste (ageusia) and change in taste (dysgeusia) are emerging symptoms. Gl symptoms (diarrhea, nausea, vomiting) with or without respiratory symptoms are reported in significant number of CoVID-19 patients. **There has been conflicting evidence regarding the utility of antimalarial agents such as hydroxychloroquine ${ }^{17}$ and the decision to utilize such therapy should take into account potential adverse effects such as ventricular arrythmias and QT prolongation common pre-existing conditions in SCD patients. Importantly, a large majority of Sickle cell disease (SCD) patients are of African descent and are at risk of drug-induced hemolysis due to concomitant G6PD deficiency. Use of hydroxychloroquine has also been associated with significant methemoglobinemia in case reports. ${ }^{18}$ With the current lack of evidence on associated risks and complications, registries to capture global information on COVID-19 SCD cases have been established: https://covidsicklecell.org/http://eurobloodnet.eu/news/99/covid-19-infection-and-redblood-cell-disorders. The sickle cell community can also obtain guidance on the management of individuals with SCD on the ASH website: https://www.hematology.org/covid-19/covid-19-and-sickle-cell-disease. ACS: acute coronary syndrome; CBC: complete blood count; CT: computed tomography.

on hydroxychloroquine and received exchange transfusion which reduced the $\mathrm{Hb} \mathrm{S}$ level from $87.1 \%$ to $18.1 \%$. He made a full recovery and was discharged after 16 days in the hospital.

The Amsterdam University Medical Centers reported two Hb SS patients who presented with typical acute sickle pain with no accompanying flu-like symptoms. ${ }^{8}$ A 24year-old male presented with acute thoracic pain in the absence of fever or dyspnea; throat and nose swabs were negative for SARS-CoV-2. Computed tomography (CT) imaging revealed bilateral pulmonary infiltrates and the patient received the presumptive diagnosis of a vasoocclusive crisis (VOC) complicated by ACS. He received antibiotic therapy and was discharged home only to return 24 hours later with increasing pain, dyspnea, and fever. Repeat chest imaging demonstrated progression of infiltrates but radiologic findings were not consistent with COVID-19 pneumonia. A repeat PCR performed on a sputum sample was positive for SARS-CoV-2. The patient received appropriate supportive treatment and had an uneventful recovery. Patient 2, a 20 -year-old female presented with an acute pain crisis. She developed transient hypoxia and subsequent SARS-CoV-2 testing was positive. Although CT imaging of the chest did not demonstrate pulmonary abnormalities, she was hospitalized for pain management and never developed respiratory symptoms or fever. We have not been informed if these patients were on hydroxyurea or regular blood transfusion programs.

Although anecdotal, a few important themes emerge from these cases - that COVID-19 might trigger a VOC without the accompanying respiratory manifestations of COVID-19, that ACS is not as common a complication as feared, and that fever was notably absent at presentation in some SCD patients, ${ }^{6-8}$ a feature also noted among 5,700 patients admitted with COVID-19 in New York City. ${ }^{9}$

\section{Are patients with SCD at greater risk for serious illness secondary to COVID-19?}

Data from non-SCD cohorts have demonstrated that advanced age and the presence of medical co-morbidities including cardiovascular disease, hypertension, diabetes mellitus, and pre-existing lung disease place individuals at higher risk for developing severe complications as a result of COVID-19, including catastrophic acute hypoxic respiratory failure. ${ }^{9,10}$ Critically ill patients can develop a cytokine storm, progressive endothelial activation with associated risk of micro- and macrothrombi, and disseminated intravascular coagulation (DIC) resulting in multi-organ failure. Marked elevations in D-dimer and prothrombin with a reduction in fibrinogen levels herald a worse prognosis and heightened risk of death in COVID-19 patients. ${ }^{11}$

Patients with SCD, in particular older adults, often have multiple comorbidities with progressive renal insufficiency, hypertension, and chronic lung disease including pulmonary hypertension. ${ }^{12}$ Viral infection can trigger acute vaso-occlusive crises, including ACS which is associated with high mortality rates. ${ }^{13}$ In this setting of multi-organ dysfunction, in particular chronic lung damage, COVID-19 could easily trigger ACS and multi-organ failure. 
Underlying endothelial dysfunction and abnormal expression of procoagulants such as tissue factor could also place SCD patients at a greater risk of thromboinflammation and thrombotic events if infected with SARS-CoV-2. ${ }^{14}$ Data from the US have shown higher rates of symptomatic infection requiring hospitalization and death in patients of African American and Hispanic ethnicity highlighting racial health disparities which impact the SCD community. ${ }^{15}$

Importantly, COVID-19 directed therapies such as antimalarial agents (hydroxychloroquine or chloroquine) may confer additional risks and must be considered carefully given conflicting data regarding effectiveness. ${ }^{16,17}$ One well known adverse effect is methemoglobinemia, particularly in individuals with concomitant G6PD deficiency. Over a 4week period in a single health system, eight COVID-19 patients who were treated with hydroxychloroquine developed methemoglobinemia which was significant in three patients necessitating treatment with methylene blue. ${ }^{18} \mathrm{Of}$ note, 1 of the 3 patients who developed acute hemolysis was found to have a new diagnosis of G6PD deficiency. In this respect, hydroxychloroquine treatment presents additional risks in SCD patients in whom concomitant G6PD deficiency is not uncommon; in addition to other adverse cardiac effects including OT prolongation and ventricular arrythmias.

\section{Strategies to optimize management of SCD patients during the COVID-19 pandemic}

Hematologists caring for SCD patients have transitioned to telemedicine to reduce unnecessary exposure to SARSCoV-2 in healthcare settings. Given asymptomatic transmission, healthcare providers should be vigilant in educating patients regarding social isolation practices in their geographic area, hand hygiene, and precautions when in public settings. Patients should be encouraged to be adherent to disease modifying therapies such as hydroxyurea to reduce the frequency of VOC episodes requiring medical attention. Laboratory monitoring for hydroxyurea and iron chelating drugs may need to be done less frequently, and when feasible, medications should be mailed or delivered to the home. Routine visits to the clinic or hospital should be avoided unless phone triage indicates acute symptoms requiring medical evaluation. Some individuals may be able to manage uncomplicated pain crises at home with optimization of oral opioid regimens and close supervision from healthcare providers. Clinical teams should develop dedicated care pathways including phone screening to assess for COVID-19 symptoms prior to a scheduled visit, clinical screening before physical entrance including temperature measurement and symptom assessment, physical distancing in waiting areas, as well as treatment in COVID-19 free clinical areas or isolation rooms. Cross-coverage of providers in the inpatient and outpatient setting should be limited and rotating clinical team schedules are encouraged to reduce the asymptomatic spread of SARS-CoV-2.

\section{Strategies to decrease blood utilization in the setting of blood shortages}

In the pandemic setting, there is a risk of severe blood shortages due not only to a decrease in donor participation but a decrease in personnel to collect and process blood. Regular blood transfusion is standard therapy for patients who have suffered an overt stroke as this offers the greatest protection from recurrence of further strokes; such patients should continue to receive transfusions in the presence of adequate blood supply. Patients with abnormal transcranial doppler (TCD) measurements may be eligible for transition to hydroxyurea therapy as per TWiTCH trial criteria for the prevention of a primary stroke. ${ }^{19,20}$ In order to preempt the possibility of blood supply interruption, it has been suggested that all children on blood transfusion therapy for primary and secondary stroke prevention should be started on low-dose hydroxyurea (HU) therapy (fixed $10 \mathrm{mg} / \mathrm{kg} /$ day). ${ }^{21}$ Dose escalation of $\mathrm{HU}$ requires frequent laboratory monitoring of peripheral blood counts which may be undesirable in areas with high rates of community spread. DeBaun emphasizes that there can only be advantages in initiating low-dose HU therapy for patients on transfusion programs for stroke prevention - low-dose HU has a minimal risk of myelosuppression, starting low-dose $\mathrm{HU}$ will decrease the lag time for clinical benefits if transfusions are suspended, and low-dose HU confers additional clinical benefits, such as reducing the frequency of $\mathrm{VOC}$ and ACS. Another approach to conserve blood during the COVID-19 pandemic is to dose-escalate hydroxyurea as this may reduce transfusion needs in SCD patients with a history of stroke. ${ }^{22}$ Outside stroke prevention, transfusion hemoglobin thresholds may be relaxed in patients without cardiopulmonary comorbidities in the absence of acute symptoms or organ dysfunction secondary to anemia. Simple blood transfusions may be substituted for exchange regimens and $\mathrm{Hb} \mathrm{S}$ goals could also be relaxed (e.g., $\mathrm{Hb} \mathrm{S}$ of $40 \%$ instead of $30 \%$ ). ${ }^{20}$ Routine pre-transfusion laboratory work should be performed on the day of a scheduled transfusion to reduce unnecessary exposure to healthcare settings. Advanced planning for patients with extensive alloimmunization is necessary given that matched blood products may be difficult to source; transfusion of the least incompatible blood product with rituximab prophylaxis may be considered in emergency situations due to life-threatening anemia. ${ }^{23} \mathrm{~A}$ triage of managing SCD patients in the COVID-19 pandemic setting is proposed in Figure 1.

\section{Conclusions}

Many unknown factors remain when considering the impact of COVID-19 in SCD patients. From published case reports, it is not clear if SCD increases the risk of SARS-CoV-2 infection. What is clear, is that fever is not always a feature, acute pain is a common presentation and COVID-19 can induce ACS but patients can recover fully with adequate supportive care. Importantly, lifesaving measures including mechanical ventilation should not be withheld from patients with SCD in the midst of this pandemic.

\section{References}

1. Fauci AS, Lane HC, Redfield RR. Covid-19 - Navigating the uncharted. N Engl J Med. 2020;382(13):1268-1269

2. Organization WH. Coronavirus disease (COVID-19) situation summary. 2020 [cited; Available from: https://www.who.int/emergencies/diseases/novel-coronavirus-2019/situation-reports]. 
3. Chakravorthy S. COVID-19 in patients with sickle cell disease - a case series from a UK tertiary hospital. Haematologica. 2020;105(11): 2691-2693.

4. Frater JL, Zini G, d'Onofrio G, Rogers HJ. COVID-19 and the clinical hematology laboratory. Int J Lab Hematol. 2020;42(1):11-18.

5. Telfer P. Real-time national survey of COVID-19 in hemoglobinopathy and rare inherited anemia patients. Haematologica. 2020;105(11):2651-2654.

6. Hussain FA, Njoku FU, Saraf SL, Molokie RE, Gordeuk VR, Han J. COVID-19 infection in patients with sickle cell disease. $\mathrm{Br} J$ Haematol. 2020;189(5):851-852.

7. Beerkens F, John M, Puliafito B, Corbett V, Edwards C, Tremblay D. COVID-19 pneumonia as a cause of acute chest syndrome in an adult sickle cell patient. Am J Hematol. 2020;95(7):E154-E156.

8. Nur E, Gaartman AE, van Tuijn CFJ, Tang MW, Biemond BJ. Vasoocclusive crisis and acute chest syndrome in sickle cell disease due to 2019 novel coronavirus disease (COVID-19). Am J Hematol. 2020;95 (6):725-726.

9. Richardson S, Hirsch JS, Narasimhan M, et al. Presenting characteristics, comorbidities, and outcomes among 5700 patients hospitalized with COVID-19 in the New York city area. JAMA. 2020;323(20):2052-2059

10. Zhou F, Yu T, Du R, et al. Clinical course and risk factors for mortality of adult inpatients with COVID-19 in Wuhan, China: a retrospective cohort study. Lancet. 2020;395(10229):1054-1062

11. Wu C, Chen X, Cai Y, et al. Risk factors associated with acute respiratory distress syndrome and death in patients with Coronavirus disease 2019 pneumonia in Wuhan, China. JAMA Intern Med. 2020;180(7):934-943.

12. Shet AS, Thein SL. A growing population of older adults with sickle cell disease. Clin Geriatr Med. 2019;35(3):349-367.

13. Vichinsky EP, Neumayr LD, Earles AN, et al. Causes and outcomes of the acute chest syndrome in sickle cell disease. National Acute Chest Syndrome Study Group. N Engl J Med. 2000;342(25):1855-1865.

14. Connors JM, Levy JH. Thromboinflammation and the hypercoagula- bility of COVID-19. J Thromb Haemost. 2020;18(7):1559-1561.

15. (MMWR). CMaMWR. Hospitalization rates and characteristics of patients hospitalized with laboratory-confirmed Coronavirus disease 2019 - COVID-NET, 14 States, March 1-30, 2020. 2020 [cited; Available from: https://www.cdc.gov/mmwr].

16. Mehra MR, Desai SS, Ruschitzka F, Patel AN. Hydroxychloroquine or chloroquine with or without a macrolide for treatment of COVID-19: a multinational registry analysis. Lancet. 2020;S01406736(20):31180-31186.

17. Yazdany J, Kim AHJ. Use of Hydroxychloroquine and chloroquine during the COVID-19 pandemic: what every clinician should know. Ann Intern Med. 2020;172(11):754-755.

18. Naymagon L, Berwick S, Kessler A, Lancman G, Gidwani U, Troy K The emergence of methemoglobinemia amidst the COVID-19 pandemic. Am J Hematol. 2020;95(8):E196-E197.

19. Ware RE, Davis BR, Schultz WH, et al. Hydroxycarbamide versus chronic transfusion for maintenance of transcranial doppler flow velocities in children with sickle cell anaemia-TCD With Transfusions Changing to Hydroxyurea (TWiTCH): a multicentre, open-label, phase 3, non-inferiority trial. Lancet. 2016;387 (10019):661-670.

20. America SCDAo. Sickle cell disease and COVID-19: provider advisory. 2020 [cited; Available from: https://www.sicklecelldisease.org /files/sites/181/2020/03/4.10.20-MARAC-SCDAA-PROVIDERADVISORY-4-10-2020].

21. DeBaun MR. Initiating adjunct low dose-hydroxyurea therapy for stroke prevention in children with SCA during the COVID-19 pandemic. Blood. 2020;135(22):1997-1999.

22. Nickel RS, Margulies S, Frazer B, Luban NLC, Webb J. Combination dose-escalated hydroxyurea and transfusion: an approach to conserve blood during the COVID-19 pandemic. Blood. 2020;135(25): 2320-2322.

23. Chou ST, Alsawas M, Fasano RM, et al. American Society of Hematology 2020 guidelines for sickle cell disease: transfusion support. Blood Adv. 2020;4(2):327-355.

\section{A CD205-directed antibody drug conjugate - lymphoma precision oncology or sophisticated chemotherapy?}

\section{Damian T. Rieke ${ }^{1,2}$ and Ulrich Keller ${ }^{1,3}$}

${ }^{1}$ Department of Hematology, Oncology and Tumor Immunology, Campus Benjamin Franklin, Charité - Universitätsmedizin Berlin, 12203 Berlin; ${ }^{2}$ Berlin Institute of Health (BIH), 10178 Berlin and ${ }^{3}$ Max-Delbrück-Center for Molecular Medicine, 13092 Berlin, Germany

E-mail: ULRICH KELLER - ulrich.keller@charite.de

doi:10.3324/haematol.2020.261073

$\mathrm{P}$ recision oncology is expected to improve outcome of patients with malignant diseases by taking into account individual variability. ${ }^{1}$ This approach is strictly linked to the availability of a targeted treatment the efficacy of which depends on the presence of a molecular alteration, i.e., a predictive biomarker. This concept has been shown to be highly successful in well-defined subgroups of patients and has led to the histology-agnostic approval of drugs in solid tumors. ${ }^{2}$ Biomarker-stratified treatment has become first-line treatment in several solid tumors, such as non-small cell lung cancer. In many hematopoietic malignancies, including B-cell lymphomas, comparably higher cure rates and more treatment options have led to a more prognosis-oriented stratification of treatment. Here, prognostic biomarkers help to adjust treatment intensity to a cohort risk assessment. ${ }^{3}$ Together with improved prognostication of patients, a more refined diagnosis also helps with better treatment allocation.
Therefore, diagnostic biomarkers will help with the identification of defined disease subgroups. ${ }^{4}$ This might also correspond to differential outcome and/or response to treatment, and can therefore overlap with predictive and/or prognostic markers.

However, despite numerous advances in the understanding of cancer heterogeneity, not all diagnostic or prognostic stratifications will ultimately impact treatment and a number of patients will eventually have disease recurrence or progression. Therefore, the identification of novel treatment strategies is urgently required. The development of additional predictive biomarkers and corresponding drugs promises to improve outcome and limit toxicity. This advancement of precision oncology can be achieved in at least two ways: (i) the identification of the right treatment for given patients (as often tried in umbrella or unstratified precision oncology trials); ${ }^{5}$ or (ii) the identification of the right patient for a given treatment (as 